\title{
Impact of Post-operative Complications after Cardiac Surgery on Long-term Survival
}

\author{
Siddharth Pahwa ${ }^{1}$, Annalisa Bernabei ${ }^{1}$, Hartzell Schaff ${ }^{1}$, John Stulak ${ }^{2}$, Kevin Greason ${ }^{3}$, \\ Alberto Pochettino ${ }^{1}$, Richard Daly ${ }^{1}$, Joseph Dearani ${ }^{1}$, Gabor Bagameri ${ }^{1}$, Katherine King ${ }^{1}$, \\ Jason Viehman ${ }^{1}$, and Juan A. Crestanello ${ }^{1}$ \\ ${ }^{1}$ Mayo Clinic \\ ${ }^{2}$ Mayo Clinc \\ ${ }^{3}$ MAYO CLINIC
}

May 18, 2020

\begin{abstract}
Background - The impact of post-operative complications on long-term survival is not well characterized. We sought to study the prevalence of post-operative complications after cardiac surgery and their impact on long-term survival. Methods - Operative survivors $(\mathrm{n}=26,221)$ who underwent coronary artery bypass grafting (CABG) $(\mathrm{n}=13054,49.8 \%)$, valve surgery $(\mathrm{n}=8667,33.1 \%)$ or combined CABG and valve surgery $(n=4500,17.2 \%)$ from 1993 to 2019 were included in the study. Records were reviewed for post-operative complications and long-term survival. The associations between post-operative complications and survival were assessed using a Cox-proportional model. Results - Complications occurred in 17,463 (66.6\%) of 26,221 operative survivors. A total of 17 post-operative complications were analyzed. Post-operative blood product use was the commonest $(\mathrm{n}=12397,47.3 \%)$, followed by atrial fibrillation $(\mathrm{n}=8399,32.0 \%)$, prolonged ventilation $(\mathrm{n}=2336,8.9 \%)$, renal failure $(\mathrm{n}=870,3.3 \%)$, re-operation for bleeding $(\mathrm{n}=859,3.3 \%)$ and pacemaker/ICD insertion $(\mathrm{n}=795,3.0 \%)$. Stroke (HR 1.55, 95\%CI 1.36-1.77), renal failure (HR $1.45,95 \%$ CI 1.33-1.58) anticoagulant-related events (HR 1.26, 95\%CI 1.02-1.56) and pneumonia (HR 1.23, 95\%CI 1.11-1.36) had the strongest impact on long-term survival. Long-term survival decreased as the number of post-operative complications increased. Conclusions - Post-operative complications after cardiac surgery significantly impact outcomes that extend beyond the post-operative period. The presence, number and type of post-operative complications adversely impact long-term survival. Stroke, renal failure, anticoagulant-related events and pneumonia are particularly associated with poor long-term survival.
\end{abstract}

\section{Hosted file}

Submission draft.DOCX available at https://authorea.com/users/322987/articles/451948-impactof-post-operative-complications-after-cardiac-surgery-on-long-term-survival

\section{Hosted file}

Table 1.docx available at https://authorea.com/users/322987/articles/451948-impact-of-postoperative-complications-after-cardiac-surgery-on-long-term-survival

\section{Hosted file}

Table 2.docx available at https://authorea.com/users/322987/articles/451948-impact-of-postoperative-complications-after-cardiac-surgery-on-long-term-survival

\section{Hosted file}

Table 3.docx available at https://authorea.com/users/322987/articles/451948-impact-of-postoperative-complications-after-cardiac-surgery-on-long-term-survival 


\section{Hosted file}

Table 4.docx available at https://authorea.com/users/322987/articles/451948-impact-of-postoperative-complications-after-cardiac-surgery-on-long-term-survival

\section{Hosted file}

Table 5.docx available at https://authorea.com/users/322987/articles/451948-impact-of-postoperative-complications-after-cardiac-surgery-on-long-term-survival

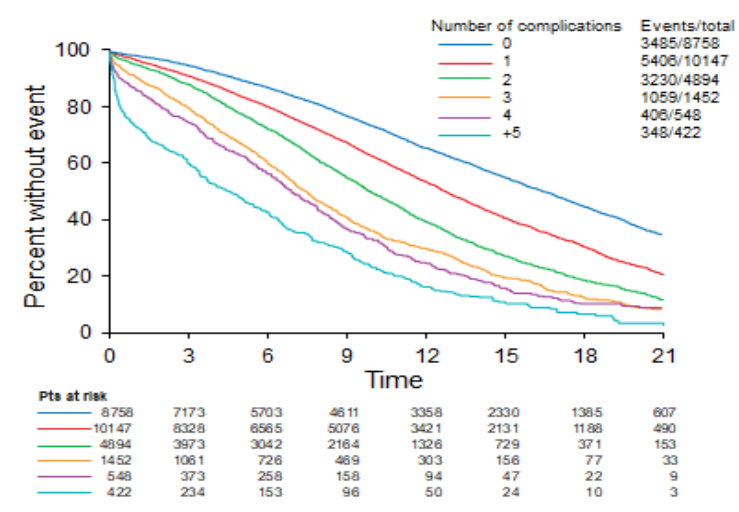


Monday, January 27, 2020 01:51:16 PM 1
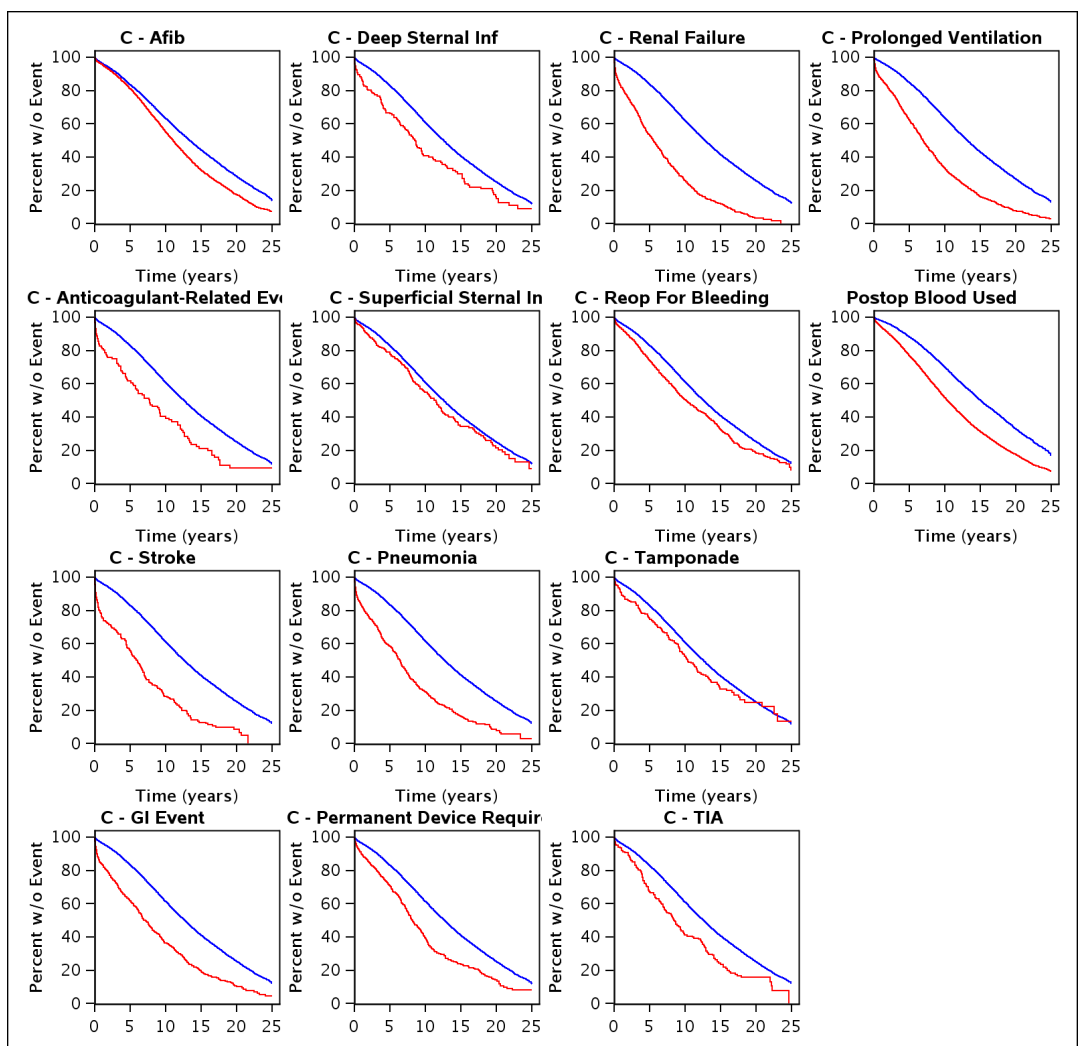

C - Permanent Device Requir

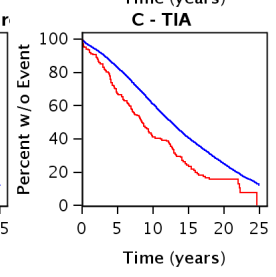

